\title{
Dephasing mechanisms of optical transitions in rare-earth-doped transparent ceramics
}

\author{
Nathalie Kunkel,,${ }^{1,2, *}$ John Bartholomew, ${ }^{1, \dagger}$ Sacha Welinski, ${ }^{1}$ Alban Ferrier,${ }^{1,3}$ Akio Ikesue, ${ }^{4}$ and Philippe Goldner ${ }^{1}$ \\ ${ }^{1}$ PSL Research University, Chimie ParisTech, CNRS, Institut de Recherche de Chimie Paris, \\ 11 rue Pierre et Marie Curie, 75005 Paris, France \\ ${ }^{2}$ Chair of Inorganic Chemistry with Focus on Novel Materials, Technical University of Munich, \\ Lichtenbergstrasse 4, 85747 Garching, Germany \\ ${ }^{3}$ Sorbonnes Universités, UPMC Univ Paris 06, 75005 Paris, France \\ ${ }^{4}$ World Laboratory, Mutsuno, Atsuta-ku, Nagoya 456-0023, Japan
}

(Received 15 August 2016; revised manuscript received 7 October 2016; published 1 November 2016)

\begin{abstract}
We identify and analyze dephasing mechanisms that broaden the optical transitions of rare-earth ions in randomly oriented transparent ceramics. The study examines the narrow ${ }^{7} F_{0} \leftrightarrow{ }^{5} D_{0}$ transition of $\mathrm{Eu}^{3+}$ dopants in a series of $\mathrm{Y}_{2} \mathrm{O}_{3}$ ceramic samples prepared under varying conditions. We characterize the temperature and magnetic field dependence of the homogeneous linewidth, as well as long-term spectral diffusion on time scales up to $1 \mathrm{~s}$. The results highlight significant differences between samples with differing thermal treatments and $\mathrm{Zr}^{4+}$ additive concentrations. In particular, several distinct magnetic interactions from defect centers are observed, which are clearly distinguished from the broadening due to interactions with two-level systems and phonons. By minimizing the broadening due to the different defect centers, linewidths of the order of $4 \mathrm{kHz}$ are achieved for all samples. The linewidths are limited by temperature-dependent interactions and by an interaction that is yet to be identified. Although the homogeneous linewidth can be narrowed further in these ceramic samples, the broadening is now comparable to the linewidths achieved in rare-earth-ion-doped single crystals. Thus, this work emphasizes the usefulness of studying ceramics to gain insights into dephasing mechanisms relevant to single crystals and suggests that ceramics may be an interesting alternative for applications in classical and quantum information processing.
\end{abstract}

DOI: 10.1103/PhysRevB.94.184301

\section{INTRODUCTION}

Materials containing trivalent rare-earth ions are promising candidates for spectral hole burning devices and quantum memories [1,2]. Their possible applications include radio frequency spectral analysis [3], laser stabilization [4], enhanced ultrasound tomography [5], signal processing, and quantum memory devices $[1,6]$. In all of these, a key element is the fact that trivalent rare-earth ions possess extremely narrow optical homogeneous linewidths [7,8]. For example, in highquality single crystals homogeneous linewidths approaching the radiative lifetime $\left(T_{1}\right)$ limit were found for several rareearth ions, including, in $\mathrm{Y}_{2} \mathrm{SiO}_{5}, 122 \mathrm{~Hz}$ for $\mathrm{Eu}^{3+}$ [9], $3.5 \mathrm{kHz}$ for $\mathrm{Nd}^{3+}$ [10], $73 \mathrm{~Hz}$ for $\mathrm{Er}^{3+}$ [8], $1 \mathrm{kHz}$ for $\mathrm{Pr}^{3+}$ [11], $3 \mathrm{kHz}$ in $\mathrm{Tm}^{3+}$-doped $\mathrm{Y}_{3} \mathrm{Al}_{5} \mathrm{O}_{12}$ at $1.5 \mathrm{~K}$ [12], and $600 \mathrm{~Hz}$ in $\mathrm{Tm}^{3+}$-doped $\mathrm{Y}_{3} \mathrm{Ga}_{5} \mathrm{O}_{12}$ [13]. Narrow homogeneous linewidths correspond to long coherence times $T_{2}$ and weak dephasing processes. Therefore, designing and preparing materials with little excess dephasing compared to the $T_{1}$ limit is an essential component in realizing high-performance rare-earth technology. To progress toward this aim, detailed investigations of dephasing processes in single crystals are invaluable (for example, Refs. [13-15]). Similarly, studies of dephasing mechanisms in glasses (see Refs. [16] and [17]) have also provided insight into dynamics that contribute significantly to the homogeneous linewidth of ions in crystals.

\footnotetext{
*Corresponding author: nathalie.kunkel@lrz.tu-muenchen.de

${ }^{\dagger}$ Present address: T. J. Watson Laboratory of Applied Physics, California Institute of Technology, 1200 E. California Blvd., Pasadena, CA 91125, USA.
}

The analysis of dephasing interactions in transparent ceramics provides information from an intermediate regime between glasses and single crystals. This information has the potential to inform future materials development strategies. One advantage of studying ceramics is that for many materials it is easier to control the growth and material properties of ceramics compared to single-crystal counterparts. As a result, systematic studies of different growth conditions can be conducted relatively quickly and inexpensively. In addition, transparent ceramics possessing narrow, homogeneous linewidths are appealing in their own right for technological applications [18]. In particular, transparent ceramics offer a larger flexibility in volume, shape, and composition than single crystals. Materials that have already been produced in the form of transparent ceramics include $\mathrm{Eu}^{3+}: \mathrm{Y}_{2} \mathrm{O}_{3}[19], \mathrm{Nd}^{3+}: \mathrm{Y}_{3} \mathrm{Al}_{5} \mathrm{O}_{12}$ [20], $\mathrm{Nd}^{3+}: \mathrm{Sc}_{2} \mathrm{O}_{3}$ [21], $\mathrm{Nd}^{3+}: \mathrm{Y}_{3} \mathrm{ScAl}_{4} \mathrm{O}_{12}$ [22], $\mathrm{Nd}^{3+}: \mathrm{Lu}_{2} \mathrm{O}_{3}$ [23], $\mathrm{Pr}^{3+}: \mathrm{Lu}_{3} \mathrm{Al}_{5} \mathrm{O}_{12}$ [24], and $\mathrm{Ba}(\mathrm{MgZr}, \mathrm{Ta}) \mathrm{O}_{3}$ [25].

A material that is extremely well suited for a detailed study of dephasing processes in transparent ceramics is $\mathrm{Eu}^{3+}$. doped $\mathrm{Y}_{2} \mathrm{O}_{3}$. In a high-quality single crystal, this material exhibits minimal additional dephasing compared to the $T_{1}$ limit and is well characterized [26,27]. Although a linewidth of $290 \mathrm{~Hz}$ has been observed [26], consistently achieving the required crystalline quality for such optical performance has been elusive. This is highlighted by the studies of different crystal growth techniques that showed that the homogeneous linewidth can vary from $2.4-42 \mathrm{kHz}$ in some crystalline fibers $[28,29]$. In addition to single-crystal studies, $\mathrm{Eu}^{3+}: \mathrm{Y}_{2} \mathrm{O}_{3}$ has also been the subject of several studies examining the dephasing processes in nanoparticles [30,31]. Therefore, there exists a base of knowledge to draw upon for this material as well as a largely unexplained variation in achievable 
homogeneous linewidths. Our previous investigations into the optical properties of $\mathrm{Eu}^{3+}: \mathrm{Y}_{2} \mathrm{O}_{3}$ transparent ceramics $[18,19]$ measured homogeneous linewidths as narrow as $10 \mathrm{kHz}$. This enables a high-resolution study of dephasing mechanisms.

In the present work we characterize the contributions of different dephasing mechanisms in $\mathrm{Eu}^{3+}: \mathrm{Y}_{2} \mathrm{O}_{3}$ transparent ceramic samples. We have studied the temperature dependence, magnetic field dependence, and spectral diffusion behavior of the optical linewidth in samples that vary in terms of the presence or absence of the additive $\mathrm{ZrO}_{2}$ and in their thermal annealing treatment. Our results demonstrate that studying ceramics can be useful in gaining insights into dephasing mechanisms relevant to single crystals and suggest that ceramics may be interesting materials for applications in classical and quantum information processing.

\section{MATERIAL AND EXPERIMENTAL DETAILS}

$\mathrm{Y}_{2} \mathrm{O}_{3}$ crystallizes in the rare-earth sesquioxide C-type structure $(I a \overline{3})$ and has two cation sites with $C_{2}$ and $S_{6}$ symmetry, respectively [32]. The optical experiments were carried out on the $C_{2}$ site, because the ${ }^{7} F_{0}-{ }^{5} D_{0}$ transition has a much greater oscillator strength than in the centrosymmetric site. Transparent ceramics were prepared from high-purity $\mathrm{Y}_{2} \mathrm{O}_{3}(99.999 \%)$ and $\mathrm{Eu}_{2} \mathrm{O}_{3}(99.99 \%)$ nanopowders according to the process described in detail in Refs. [18] and [19]. A sintering aid $\left(0.5 \% \mathrm{ZrO}_{2}\right)$ was added to some samples. All samples were sintered under vacuum and then hot isostatically pressed (HIP) at $1600^{\circ} \mathrm{C}$ and $147 \mathrm{MPa}$ for $2 \mathrm{~h}$. After the HIP, some samples were annealed under air at $1100^{\circ} \mathrm{C}$ (post-HIP annealing). The final diameter and thickness of all samples were $12 \mathrm{~mm}$ and $6 \mathrm{~mm}$, respectively. The grain sizes were estimated by optical microscopy to be around $1 \mu \mathrm{m}$.

From previous electron paramagnetic resonance (EPR) and (thermally stimulated) luminescence studies [33] it is known that, depending on the synthesis conditions, different defect centers are present. In samples without post-HIP annealing the presence of $F$ centers (two electrons on an oxygen vacancy) is suggested based on the presence of a thermoluminescence glow peak and short-lived luminescence. Such F centers are likely to be created during the vacuum treatment. Furthermore, small amounts of $\mathrm{F}^{+}$centers (one electron on an oxygen vacancy) and $\mathrm{O}_{2}^{-}$ions were detected by EPR. In samples with post-HIP annealing, the F-center signal disappears but there are significant signals arising from the presence of $\mathrm{F}^{+}$centers and $\mathrm{O}_{2}^{-}$ions, respectively.

Optical experiments were carried out on the ${ }^{7} F_{0} \rightarrow{ }^{5} D_{0}$ transition (580.88 $\mathrm{nm}$ in vacuum), which has an inhomogeneous linewidth of about $20 \mathrm{GHz}$ in the samples without additives and about $100 \mathrm{GHz}$ in the ones with additives [33]. Homogeneous linewidths were measured by photon echo experiments. Two- and three-pulse photon echo measurements of the ${ }^{7} F_{0} \rightarrow{ }^{5} D_{0}$ transition (580.88 nm in vacuum) at zero magnetic field were carried out in a helium bath cryostat at temperatures between 1.7 and $15 \mathrm{~K}$ using different laser powers. Measurements in magnetic fields up to $3 \mathrm{~T}$ were taken in a helium bath cryostat with a superconducting magnet. Two-pulse photon echoes at zero field were performed using a Coherent 899-21 cw dye laser with a 1-MHz linewidth and exciting and rephasing pulses of 3.1 and $4 \mu \mathrm{s}$. The signal was gated using an acousto-optic modulator and detected with a photodiode. In order to avoid hole burning, the laser was scanning $\sim 1 \mathrm{GHz}$ in $\sim 3 \mathrm{~s}$. All other echo sequences used a Sirah Matisse 2 DR cw dye laser with a 300-kHz linewidth and heterodyne detection. For the latter case, chirped pulses over a broad spectral range were applied after each echo sequence in order to avoid hole burning effects. Three-pulse photon echo experiments used 3.1- $\mu$ s-long pulses. Except where noted otherwise, in the following text, the echo signal was averaged for between 50 and 100 shots to mitigate any contribution from the laser linewidth to the decoherence rate. In the case of heterodyne detection, the Fourier transform of the heterodyne signal was averaged to account for phase variations from one shot to the next. All two-pulse photon echo decays were found to be well fitted by a single exponential of the form $I=I_{0} \exp \left(-4 \tau / T_{2}\right)$, where $I$ is the echo intensity, $\tau$ is the delay between the exciting and rephasing pulses, and $T_{2}$ is the coherence lifetime of the transition. The corresponding homogeneous linewidth is given by $\Gamma_{h}=1 / \pi T_{2}$. In the threepulse echo sequence, the two first pulses, separated by a delay $t_{12}$, create a spectral grating. The third pulse, delayed by $T_{W}$ from the second one, is diffracted by the grating and produces an echo at a time $t_{12}+T_{W}$. For a fixed value of $T_{W}$, the echo decays as a function of $t_{12}$ were single exponential and fitted with the same expression as for the two-pulse echoes. This allowed us to determine an effective homogeneous linewidth as a function of $T_{W}, \Gamma_{\text {eff }}\left(T_{W}\right)$.

\section{RESULTS AND DISCUSSION}

\section{A. Theory}

The homogeneous linewidth $\left(\Gamma_{\mathrm{h}}\right)$ is a measure of the dynamic processes in the local environment of the activator ion. The total linewidth consists of several contributions as expressed by the following equation:

$$
\Gamma_{\mathrm{h}}=\Gamma_{\text {pop }}+\Gamma_{\text {ion-ion }}+\Gamma_{\text {ion-spin }}+\Gamma_{\text {phonon }}+\Gamma_{\mathrm{TLS}},
$$

where $\Gamma_{\text {pop }}$ is the contribution of the excited-state lifetime $T_{1}$ via the relation $\Gamma_{\text {pop }}=\frac{1}{2 \pi T_{1}}$ and has a value of about $170 \mathrm{~Hz}$ for our $\mathrm{Eu}^{3+}$-doped $\mathrm{Y}_{2} \mathrm{O}_{3}$ ceramics (excited-state lifetime $\sim 1 \mathrm{~ms}$, see Ref. [18]).

The mechanism that is responsible for the contribution $\Gamma_{\text {ion-ion }}$ is excitation-induced instantaneous spectral diffusion (ISD) [34-37]. Random frequency shifts are caused by changes in the local environments of the ions due to the change in an ion's electric dipole moment when it is optically excited to the ${ }^{5} D_{0}$ state [14]. The dephasing caused by the excitationinduced frequency shifts of the $\pi$ pulse are not rephased and hence, lead to additional broadening.

$\Gamma_{\text {ion-spin }}$ is the broadening caused by electron or nuclear spin flips that induce magnetic field fluctuations. The oxygen isotope ${ }^{16} \mathrm{O}$ (natural abundance $99.7 \%$ ) has a zero nuclear spin and the contribution of yttrium nuclear spins is known to be 200-300 Hz [27].

The broadening $\Gamma_{\text {phonon }}$ is caused by the phonon contribution. Non-Kramers ions, such as $\mathrm{Eu}^{3+}$, exhibit a $T^{7}$ behavior that is characteristic for elastic Raman scattering according to the Debye model [38]. In high-quality single crystals of $\mathrm{Eu}^{3+}: \mathrm{Y}_{2} \mathrm{O}_{3}$, the temperature dependence between 12 and $35 \mathrm{~K}$ 
was found to be in good agreement with a two-phonon Raman process [28,39].

The remaining term in Eq. (1), $\Gamma_{\mathrm{TLS}}$, is the broadening due to dynamic fluctuations due to nearly equivalent configurations in the local environment (TLS: two-level system). Such a behavior is typical for glasses [17,40] and other disordered materials $[41,42]$, and was also observed in $\mathrm{Eu}^{3+}: \mathrm{Y}_{2} \mathrm{O}_{3}$ fibers grown by laser-heated pedestal growth [28,29].

Another contribution to line broadening is spectral diffusion, which often occurs on time scales greater than $T_{2}$. Spectral diffusion has been observed in electron and nuclear spin resonance [43,44] and optical spectroscopy [14,45-48]. It occurs in the presence of time-dependent perturbations of the transition frequencies of the ions. When the transition frequencies shift over time, the homogeneous linewidth $\Gamma_{\mathrm{h}}$ appears to broaden over time, increasing the rate of decoherence. It is possible to characterize the amount of spectral diffusion on time scales that are long compared to the coherence time by using stimulated photon echo spectroscopy. Here, the echo decays are recorded as a function of pulse separation in a three-pulse photon echo sequence $[14,49]$. As mentioned in Sec. II, the delay between the first and second pulse $\left(t_{12}\right)$ was varied for a fixed delay between the second and third pulse $T_{W}$ (waiting time). The effective homogeneous linewidth $\Gamma_{\text {eff }}\left(T_{W}\right)$ was then determined from the exponential echo decay. In the case of spectral diffusion caused by spin flips and in the limit $t_{12} \rightarrow 0$, the homogeneous linewidth is given by

$$
\Gamma_{\text {eff }}\left(T_{W}\right)=\Gamma_{0}+\frac{1}{2} \Gamma_{\mathrm{SD}}\left[1-\exp \left(-R T_{W}\right)\right]
$$

where $\Gamma_{0}$ is the linewidth in the absence of spectral diffusion, $\Gamma_{\mathrm{SD}}$ the broadening due to reorientation of magnetic spins, and $R$ the spin-flip rate. A detailed derivation of this expression can be found in Ref. [14] and references therein. In the presence of TLS an additional term is added [50,51]:

$$
\Gamma_{\mathrm{eff}}\left(T_{W}\right)=\Gamma_{0}+\frac{1}{2} \Gamma_{\mathrm{SD}}\left[1-\exp \left(-R T_{W}\right)\right]+\Gamma_{\mathrm{TLS}} \log \frac{T_{W}}{T_{W}^{0}},
$$

where $T_{W}^{0}$ is a normalizing constant.

\section{B. Experimental results}

\section{Temperature dependence of the homogeneous linewidths}

In order to study the TLS and phonon contributions, the temperature dependence of the homogeneous linewidth was measured by two-pulse photon echoes. In Figs. 1(a) and 1(b) the temperature dependence is shown for samples with $0.5 \%$ $\mathrm{Eu}^{3+}$, without and with the additive $\mathrm{ZrO}_{2}$.

In all samples, the homogeneous linewidth begins to increase rapidly at temperatures above $8 \mathrm{~K}$. This increase is consistent with two-phonon Raman scattering and is well described by the term $\alpha_{\text {phonon }} T^{7}$. In the present ceramic samples, values for $\alpha_{\text {phonon }}$ close to $0.001 \mathrm{~Hz} / \mathrm{K}^{7}$ were found (see Table I). In Eu ${ }^{3+}: \mathrm{Y}_{2} \mathrm{O}_{3}$ single crystals a value of $0.0014 \mathrm{~Hz} / \mathrm{K}^{7}$ was observed [39], whereas in $\mathrm{Eu}^{3+}: \mathrm{Y}_{2} \mathrm{SiO}_{5}$ single crystals $0.0072 \mathrm{~Hz} / \mathrm{K}^{7}$ and $0.0018 \mathrm{~Hz} / \mathrm{K}^{7}$ were found for sites 1 and 2 , respectively [52]. The values measured in the ceramics are

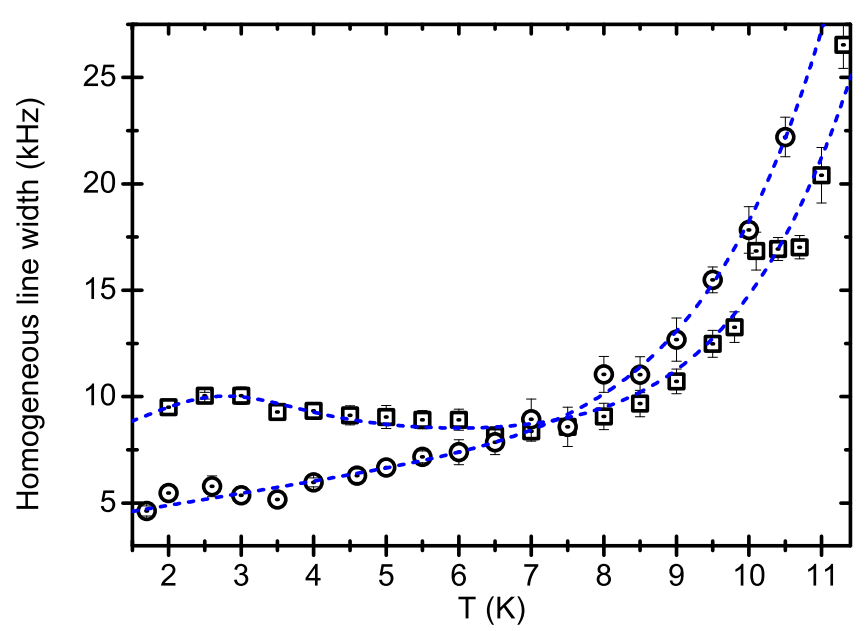

(a) $\mathrm{Eu}^{3+}: \mathrm{Y}_{2} \mathrm{O}_{3}$ without $\mathrm{ZrO}_{2}$.

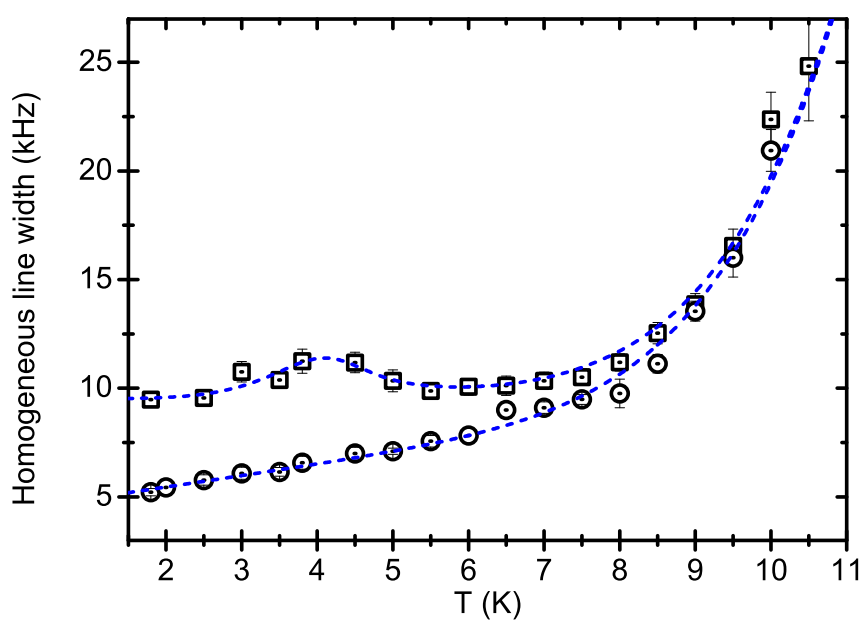

(b) $\mathrm{Eu}^{3+}: \mathrm{Y}_{2} \mathrm{O}_{3}$ with $0.5 \% \mathrm{ZrO}_{2}$.

FIG. 1. Temperature dependence of $\Gamma_{\mathrm{h}}$ for the ${ }^{7} F_{0} \rightarrow{ }^{5} D_{0}$ line in $\mathrm{Eu}^{3+}: \mathrm{Y}_{2} \mathrm{O}_{3}$ transparent ceramics with $0.5 \%$ europium, with and without post-HIP annealing, and with (b) and without (a) $\mathrm{ZrO}_{2}$. Squares: no post-HIP annealing, circles: with post-HIP annealing. Dotted lines: fit to the data with $\Gamma_{\mathrm{h}}=\Gamma_{\mathrm{h}, 0}+\Gamma_{\mathrm{ma}}+\alpha_{\text {phonon }} T^{7}$ for the sample without post-HIP annealing and $\Gamma_{\mathrm{h}}=\Gamma_{\mathrm{h}, 0}+\alpha_{\mathrm{TLS}} \mathrm{T}+$ $\alpha_{\text {phonon }} T^{7}$ for the sample with post-HIP annealing.

thus in good agreement with the two-phonon Raman model and are consistent with other $\mathrm{Eu}^{3+}$-doped crystals.

Although all samples exhibit similar linewidths at higher temperatures, there is a clear contrast in temperature dependence below $8 \mathrm{~K}$. The samples with post-HIP annealing show homogeneous linewidths at low temperature that are below $5 \mathrm{kHz}$ and a linear increase in the low-temperature region which can be attributed to TLS. To model this linear increase, we use a term $\alpha_{\mathrm{TLS}} \mathrm{T}^{n}$ with $n=1$ (see Fig. 1) and find $\alpha_{\mathrm{TLS}} \approx 0.5 \mathrm{kHz} / \mathrm{K}$. A temperature-dependent broadening of the homogeneous linewidth due to TLS in $\mathrm{Eu}^{3+}: \mathrm{Y}_{2} \mathrm{O}_{3}$ has been observed previously, both in powder and in crystalline fibers. For crystalline domains of $60 \mathrm{~nm}, \alpha_{\mathrm{TLS}} \approx 18 \mathrm{kHz} / \mathrm{K}$ was found [31], and for different fiber crystal samples, $1-15 \mathrm{kHz} / \mathrm{K}$ [29]. Thus, the influence of TLS is much weaker in the ceramics than in the crystalline fibers. Given the 
TABLE I. Summary of spectroscopic properties for $0.5 \% \mathrm{Eu}^{3+}: \mathrm{Y}_{2} \mathrm{O}_{3}$ transparent ceramics. Two-pulse photon echoes: $\Gamma_{\mathrm{h}}$ extrapolated from laser power dependence at $3.5 \mathrm{~K} ; \Gamma_{\mathrm{h}, 0}$ extrapolated from temperature dependence, $\alpha_{\mathrm{TLS}}$ from temperature dependence (a TLS exponent $n=1$ was assumed), $\alpha_{\text {phonon }}$ from temperature dependence. Stimulated photon echoes: $\Gamma_{0}$, broadening due to reorientation of magnetic spins $\Gamma_{\mathrm{SD}}$, spin-flip rate of the perturbing spins $R$, and $\Gamma_{\mathrm{TLS}}\left(T_{W}^{0}=1 \mathrm{~s}\right) . R$ was obtained for the sample with post-HIP annealing and without additive and used for modeling the other samples.

\begin{tabular}{|c|c|c|c|c|c|c|c|c|c|c|}
\hline \multicolumn{2}{|c|}{ Material } & \multirow[b]{2}{*}{$T[\mathrm{~K}]$} & \multirow[b]{2}{*}{$\Gamma_{\mathrm{h}}[\mathrm{kHz}]$} & \multicolumn{3}{|c|}{ Two-pulse photon echo } & \multicolumn{4}{|c|}{ Three-pulse photon echo } \\
\hline$\left[\mathrm{Zr}^{4+}\right](\%)$ & Ann. & & & $\Gamma_{\mathrm{h}, 0}[\mathrm{kHz}]$ & $\alpha_{\mathrm{TLS}}[\mathrm{Hz} / \mathrm{K}]$ & $\alpha_{\text {phonon }}\left[\mathrm{Hz} / \mathrm{K}^{7}\right]$ & $\Gamma_{0}[\mathrm{kHz}]$ & $\Gamma_{\mathrm{SD}}[\mathrm{kHz}]$ & $R\left[\mathrm{~s}^{-1}\right]$ & $\Gamma_{\mathrm{TLS}}[\mathrm{kHz}]$ \\
\hline- & - & 3.5 & $7.4(1)$ & - & - & - & - & - & - & - \\
\hline- & - & $1.7-12$ & - & $7.7(3)$ & - & $0.00068(4)$ & - & - & - & - \\
\hline 0.5 & - & 3.5 & $8.7(3)$ & - & - & - & - & - & - & - \\
\hline 0.5 & - & $1.7-12$ & - & $9.5(1)$ & - & $0.00102(5)$ & - & - & - & - \\
\hline- & $\mathrm{x}$ & 3.5 & $5.1(2)$ & - & - & - & - & - & - & - \\
\hline- & $\mathrm{x}$ & $1.7-12$ & - & $3.7(2)$ & $560(40)$ & $0.00088(5)$ & - & - & - & - \\
\hline- & $\mathrm{x}$ & 2 & - & - & - & - & $3.6(2)$ & $29.2(9)$ & 320 & $0.6(1)$ \\
\hline- & $\mathrm{x}$ & 3.5 & - & - & - & - & $4.3(2)$ & $40(1)$ & 1000 & $0.6(1)$ \\
\hline- & $\mathrm{x}$ & 5 & - & - & - & - & $6.1(6)$ & $48(5)$ & 3000 & $0.0(6)$ \\
\hline 0.5 & $\mathrm{x}$ & 3.5 & $5.2(1)$ & - & - & - & - & - & - & - \\
\hline 0.5 & $\mathrm{x}$ & $1.7-12$ & - & $4.4(2)$ & $520(50)$ & $0.00097(9)$ & - & - & - & - \\
\hline 0.5 & $\mathrm{x}$ & 2 & - & - & - & - & $3.8(2)$ & $12(1)$ & 320 & $0.9(1)$ \\
\hline 0.5 & $\mathrm{x}$ & 3.5 & - & - & - & - & $5.2(1)$ & $14(1)$ & 1000 & $0.9(1)$ \\
\hline 0.5 & $\mathrm{x}$ & 6 & - & - & - & - & $7.3(3)$ & $8(2)$ & 6200 & $1.8(4)$ \\
\hline
\end{tabular}

previous finding that $\mathrm{F}^{+}$and $\mathrm{O}_{2}^{-}$centers are present in these samples [33], we relate the linear temperature dependence of the homogeneous linewidth in the low-temperature region and thus, the dynamic disorder to these centers.

While the $\mathrm{Eu}^{3+}$ ions in the samples that undergo postHIP annealing possess linewidths that linearly increase with temperature, the behavior of ions in samples without postHIP annealing is clearly nonlinear. As shown in Fig. 1, the homogeneous linewidth increases to a local maximum at temperatures below $4 \mathrm{~K}$ and then decreases again for temperatures up to approximately $6 \mathrm{~K}$. The extent of the decrease is such that in the samples without the additive, the minimum linewidth is attained at a temperature between $6 \mathrm{~K}$ and $7 \mathrm{~K}$.

To the best of our knowledge, such an anomalous temperature dependence of $\Gamma_{\mathrm{h}}$ has not been previously reported for optical transitions. Although it is not possible to definitively identify the underlying cause of the local maxima in broadening, it is possible to partially characterize the likely physical mechanism. We attribute the observed behavior to a magnetic field perturbation that has a characteristic rate $R$ that varies strongly with temperature. As $R$ changes with temperature, the perturbation time scale becomes comparable to the time scale of the photon echo measurements, leading to an increase in broadening. Further increases in $R$ lead to motional averaging and a corresponding decrease in linewidth as the temperature increases up to the point where $\Gamma_{\text {phonon }}$ begins to dominate. A similarly nonlinear temperature dependence has been observed for spin transitions in the microwave regime, such as the results reported by Mims in his foundational paper on spectral diffusion [44]. The equations presented by Mims can also be applied in the optical domain and allow the behavior of the motional averaging to be modeled as a Gaussian perturbation with a temperature-dependent rate $R$ and variance $\Delta \omega$. In particular, we use Eq. (4) from Mims' work adjusted for intensity detection, such that the echo intensity $I$ at a time $2 \tau$ after the $\frac{\pi}{2}$ pulse is given by

$$
I(2 \tau)=\exp \left(-1.88 \Delta \omega[B(\tau)]^{1 / 2}\right),
$$

where

$$
\begin{aligned}
B(\tau)= & \left(1 / R^{2}\right)\{R \tau-[1-\exp (-R \tau)] \\
& \left.-0.5[1-\exp (-R \tau)]^{2}\right\},
\end{aligned}
$$

and $R$ is temperature dependent. In our work we model $R=$ $a T^{b}$ and fit $a, b$, and $\Delta \omega$ to the data. This is done by calculating an echo decay curve for values of $a, b, \Delta \omega$, and $T$ from Eqs. (4) and (5) and fitting this curve to an exponential to determine the calculated homogeneous linewidth and compare it to the experimental value.

Although this model qualitatively reproduces the observed broadening (Fig. 1), the fitting is poorly constrained due to the small number of points in the nonlinear region between 1.4 and $6 \mathrm{~K}$. Equally good fits were produced for $b$ ranging from 4 to 11 , with $a$ varying over many orders of magnitude. Despite this, the local maximum in the broadening constrains the values for $\Delta \omega$ in all cases to the order of $30 \mathrm{kHz}$.

Further investigations are needed to fully understand this broadening mechanism, but given previous materials analysis of these transparent ceramics [33] it is likely that the temperature dependence is related to the fluctuations of defect centers. One further measurement that could provide additional information is to apply rf or microwave fields that are resonant with one of the known defects during the photon echo sequence [53]. The degree of increased broadening, observed as a decrease in echo amplitude, may assist in identifying the defect or defects responsible for the anomalous temperature dependence. It seems unlikely that the behavior is caused by simple $\mathrm{F}^{+}$centers because it is apparently not 
observed in samples with post-HIP annealing. However, it still cannot be completely excluded that the presence of $\mathrm{F}$ centers in the samples without post-HIP annealing leads to a change in the flipping rate of the $\mathrm{F}^{+}$centers in these samples. It is also possible that $\mathrm{F}$-center clusters or defect clusters of $\mathrm{F}$ and $\mathrm{F}^{+}$ centers form.

\section{Magnetic field dependence of the homogeneous linewidths}

Measurements of the homogeneous linewidth dependence on an applied magnetic field for samples without post-HIP annealing show first an increase of the linewidth with increasing field strength and a subsequent decrease at higher fields (Fig. 2). The difference between the linewidth at zero field $(4.7 \mathrm{kHz})$ and the maximum linewidth is about $0.5 \mathrm{kHz}$, with the maximum linewidth situated at approximately $100 \mathrm{mT}$. The decrease of the linewidth in fields up to $2.25 \mathrm{~T}$ is about $1 \mathrm{kHz}$ and can be explained by a decrease in the dephasing induced by magnetic defects. In the limit that the flip rate of these defects is fast compared to the time scale of the echo measurements, the $\mathrm{Eu}^{3+}$ linewidth can be approximated by the expression $[54,55]$

$$
\Gamma_{\mathrm{h}}(B)=\Gamma_{0}+\Gamma_{\mathrm{d}} e^{-\beta g B / k T},
$$

where $\Gamma_{\mathrm{d}}$ is the maximal optical broadening caused by the sudden jumps between two spin states of the defects, $\beta$ is the Bohr magneton, $k$ the Boltzmann constant, $T$ the temperature, and $B$ the magnetic field. The full broadening $\Gamma_{\mathrm{d}}$ corresponds to all spins having been flipped, which at low temperature is not fully realized. This is taken into account by the the exponential factor in Eq. (6) [54]. As the magnetic field increases, the spin polarization increases, which leads to a reduced broadening of the optical line. Experimental data for fields between 0.35 and $2.25 \mathrm{~T}$ could be well fitted using $g=2$, as shown in Fig. 2 , giving $\Gamma_{0}=3.7 \mathrm{kHz}$ and $\Gamma_{\mathrm{d}}=1.8 \mathrm{kHz}$. This suggests that the centers causing the increased homogeneous linewidth in samples without post-

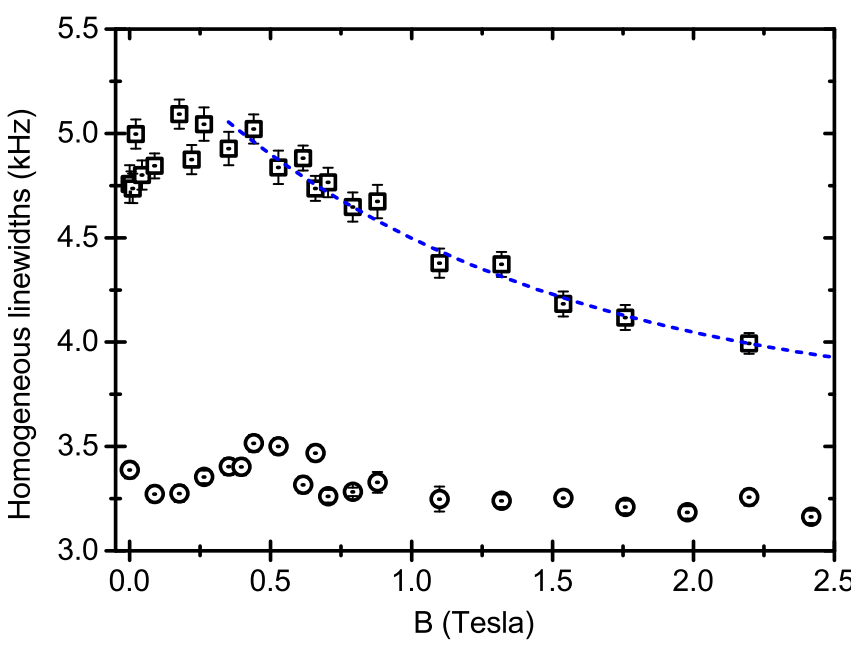

FIG. 2. Magnetic field dependence at $T=1.7 \mathrm{~K}$ of $\Gamma_{\mathrm{h}}$ for the ${ }^{7} F_{0} \rightarrow{ }^{5} D_{0}$ line in a $0.5 \% \mathrm{Eu}^{3+}: \mathrm{Y}_{2} \mathrm{O}_{3}$ transparent ceramic without (squares) and with post-HIP annealing (circles) and without $\mathrm{ZrO}_{2}$. The fit to the data for the sample without post-HIP annealing is described in the text.
HIP annealing are related to electrons trapped in oxygen vacancies. The value of $\Gamma_{0}$ extracted from the fit is $3.7 \mathrm{kHz}$, about $0.5 \mathrm{kHz}$ larger than the linewidth measured in the samples with post-HIP annealing. This could be due to centers with lower $g$ factors that are not significantly polarized even at high fields. As in the temperature-dependence results, the initial increase of linewidth with the magnetic field could be due to an increase of the flipping rate of the defect spins that at higher fields results in a motional averaging effect.

Samples with post-HIP annealing show a very small increase in the homogeneous linewidth of approximately $0.2 \mathrm{kHz}$ around $0.5 \mathrm{~T}$. The linewidth at small fields and higher fields are both close to $3.2 \mathrm{kHz}$. At low (high) fields the observed linewidths are approximately $1.5 \mathrm{kHz}(0.5 \mathrm{kHz})$ narrower than the samples without post-HIP annealing. The lack of a large magnetic field dependence indicates that the magnetic defects' concentration has been significantly reduced and is in agreement with our attribution of perturbing centers to structures involving electrons trapped in oxygen vacancies.

\section{Temperature dependence of spectral diffusion}

Stimulated photon echoes were measured at different temperatures and are shown for all types of samples (with and without post-HIP annealing, with and without $\mathrm{ZrO}_{2}$ ) in Figs. 3(a)-3(d). Especially in the samples with postHIP annealing, a significant broadening of the effective homogeneous linewidth can be observed with increasing $T_{W}$ [Figs. 3(a) and 3(c)], which is a clear sign for the presence of spectral diffusion. The increase in uncertainty for the data points taken at long $T_{W}$ is due to a decrease in the number of averages for each echo signal to a minimum of ten averages.

On short time scales, a slight linear increase is observed with respect to $\log \left(T_{W}\right)$, followed by a rapid broadening until a plateau is reached. This rapid broadening is consistent with magnetic spin-flip interactions and has been observed before, for instance, in $\mathrm{Er}^{3+}: \mathrm{Y}_{2} \mathrm{SiO}_{5}$ [14]. The observation of a slight linear increase for short waiting times (on a log scale) and the fact that the plateaus are not perfectly flat are indicative of the presence of TLS-type diffusion. The greatest level of broadening out of all the samples was observed in the samples with post-HIP annealing and without $\mathrm{ZrO}_{2}: \Gamma_{\text {eff }}<25 \mathrm{kHz}$. This result is correlated with the fact that in such samples the highest defect concentration of $\mathrm{F}^{+}$centers and $\mathrm{O}_{2}^{-}$ions has been observed [33]. Therefore, we tentatively assign the rapid broadening to an interaction with these defect center spins. We fitted the data for this sample according to the spectral diffusion model presented in Eq. (3). The fits are included in Figs. 3(a) and 3(c), and the relevant parameters are listed in Table I. The temperature-dependent parameter $R$ obtained from these fits was used to constrain the data fitting for the samples with post-HIP annealing and with $\mathrm{ZrO}_{2}$. The origin of the temperature dependence of the spin-flip rate $R$ is unclear. Under the assumption that the defect is an isolated electron spin, the significant variation in $R$ observed with zero applied field makes the dominant interaction unlikely to be direct phonon, two-phonon Raman, or mutual spin-spin interactions [56]. Two-phonon resonant Orbach processes are 


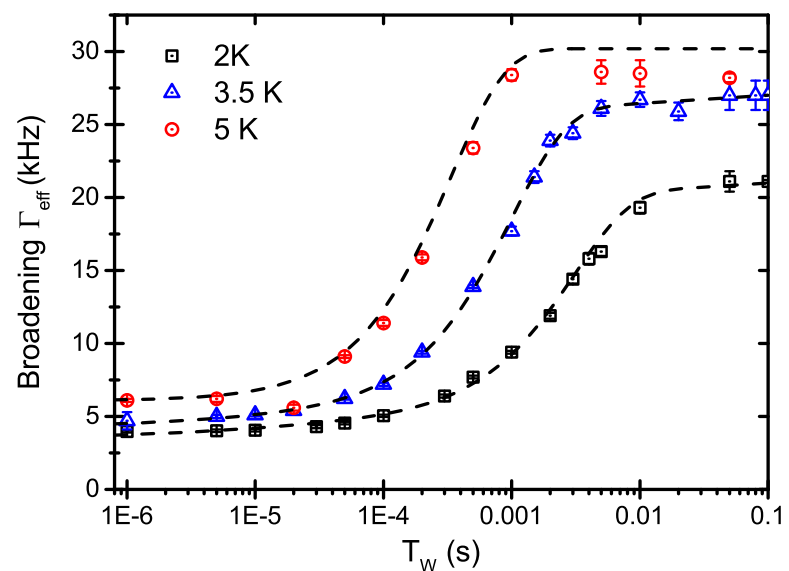

(a) $\mathrm{Eu}^{3+}: \mathrm{Y}_{2} \mathrm{O}_{3}$ without additive, with post HIP annealing.

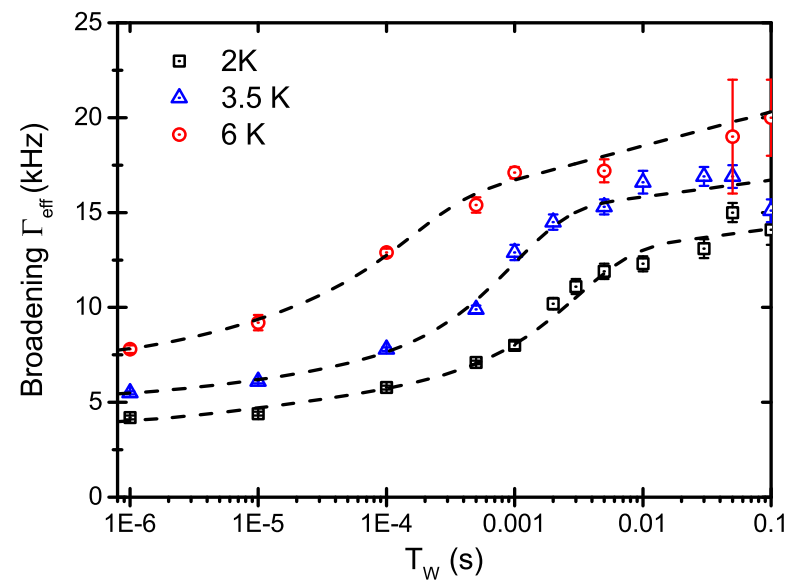

(c) $\mathrm{Eu}^{3+}: \mathrm{Y}_{2} \mathrm{O}_{3}$ with additive, with post HIP annealing.

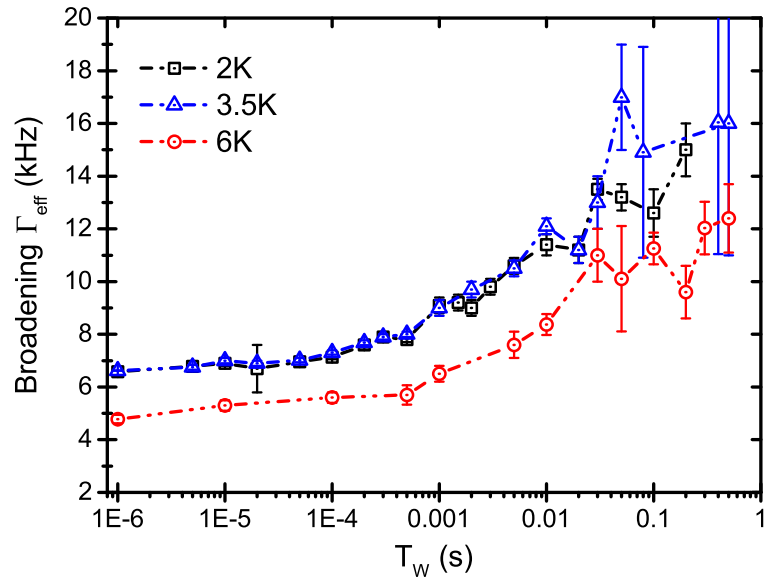

(b) $\mathrm{Eu}^{3+}: \mathrm{Y}_{2} \mathrm{O}_{3}$ without additive, without post HIP annealing.

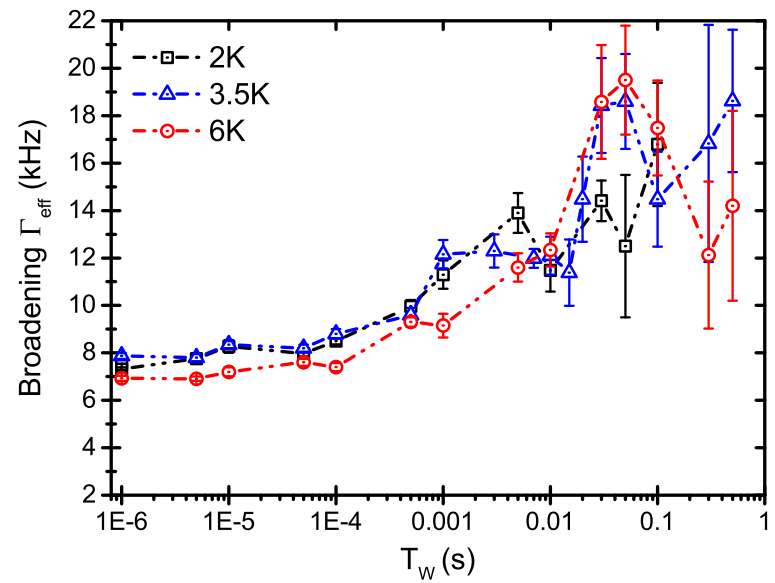

(d) $\mathrm{Eu}^{3+}: \mathrm{Y}_{2} \mathrm{O}_{3}$ with additive, without post HIP annealing.

FIG. 3. Temperature dependence of $\Gamma_{\text {eff }}$ for the ${ }^{7} F_{0} \rightarrow{ }^{5} D_{0}$ line in $\mathrm{Eu}^{3+}: \mathrm{Y}_{2} \mathrm{O}_{3}$ transparent ceramics with $0.5 \%$ europium with and without additive, and with without post-HIP annealing, obtained by stimulated photon echoes. Dotted lines: fit to the data with $\Gamma_{\text {eff }}\left(T_{W}\right)=\Gamma_{0}+$ $\frac{1}{2} \Gamma_{\mathrm{SD}}\left[1-\exp \left(-R T_{W}\right)\right]+\Gamma_{\mathrm{TLS}} \log \frac{T_{W}}{T_{W}^{0}}$, for the samples with post-HIP annealing. Samples without post-HIP annealing: dash-dotted lines are a guide to the eye.

also unlikely given that electron spin defects do not typically possess nearby crystal field levels accessible by phonons. A more detailed study of the temperature and magnetic field dependence of the spectral diffusion may clarify the origin of the spin-flip mechanism and whether models involving coupled spin defects need to be considered. With only three data points for $R$, any prediction of the value at $6 \mathrm{~K}$ was poorly constrained, but good agreement with the data was found for $R(6 \mathrm{~K})=6200$. Overall, good agreement with experimental data is obtained [Figs. 3(a) and 3(c)]. In the samples containing additives, modeling shows that $\Gamma_{\mathrm{SD}}$ is reduced. This is consistent with the decrease of both the $\mathrm{O}_{2}^{-}$ and $\mathrm{F}^{+}$center concentrations that were previously determined by EPR measurements [33]. On the other hand, the increase in $\Gamma_{\mathrm{TLS}}$ can be related to the disorder induced by $\mathrm{Zr}^{4+}$ ions. However, this seems to be a slow dephasing mechanism, as it is not apparent in two-pulse photon echo measurements (Fig. 1 and Table I).

In contrast to annealed samples, the broadening is much smaller for the samples without post-HIP annealing (approximately $10 \mathrm{kHz}$ ), as shown in Figs. 3(b) and 3(d). Furthermore, at $6 \mathrm{~K}$ the effective homogeneous linewidth for samples without post-HIP annealing becomes significantly narrower than at lower temperatures. This is in line with the observation of an anomalous temperature dependence in the two-pulse photon echoes. The stimulated echo data for samples without post-HIP annealing at higher temperatures could not be modeled using Eq. (3), indicating that the time-dependent effective broadening is not consistent with a spin-flip interaction or a TLS contribution.

\section{Summary of the dephasing contributions}

The homogeneous linewidths reported in this work are among the narrowest optical transitions observed in $\mathrm{Eu}^{3+}$. doped materials. Specifically, in the host crystal $\mathrm{Y}_{2} \mathrm{O}_{3}$, only one other sample has yielded a narrower optical transition ( $290 \mathrm{~Hz}$ achieved in the $0.3 \% \mathrm{Eu}^{3+}: \mathrm{Y}_{2} \mathrm{O}_{3}$ used in the work of Refs. [26,27,39,57]). More importantly, the spectroscopic characterization of the contributing broadening mechanisms 


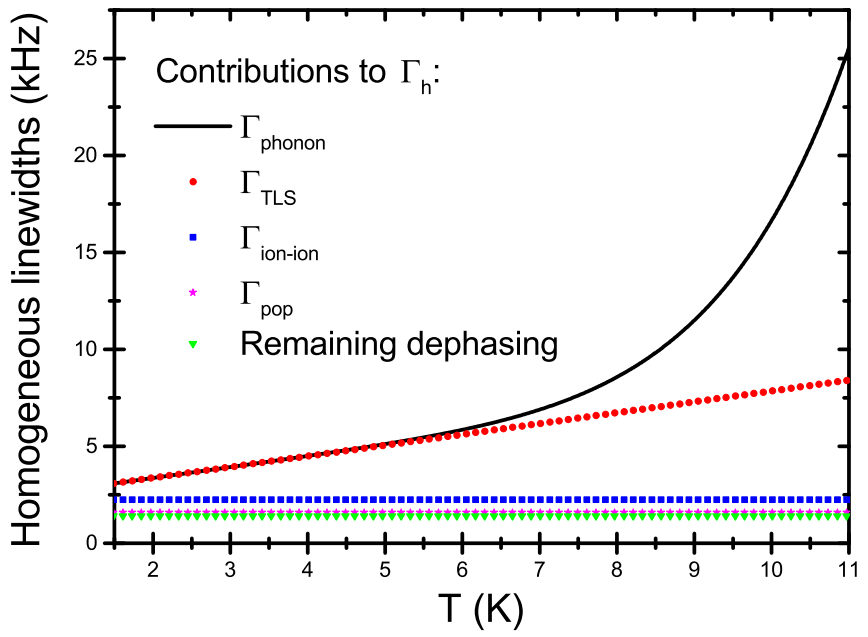

FIG. 4. Visual summary of the broadening contributions for the ${ }^{7} F_{0} \rightarrow{ }^{5} D_{0}$ line in a Eu ${ }^{3+}: \mathrm{Y}_{2} \mathrm{O}_{3}$ transparent ceramic (0.5\%) with post-HIP annealing and without $\mathrm{ZrO}_{2}$.

presented in Secs. III B1, III B2, and III B3 makes it possible to analyze to what extent polycrystalline ceramic samples can approach the linewidths achieved in bulk crystals. In doing so we discuss the feasibility of improving on the current result.

Here we present a breakdown of the broadening contributions for the $0.5 \% \mathrm{Eu}^{3+}$ sample without $\mathrm{ZrO}_{2}$ that was treated with post-HIP annealing, which yielded a $3.2 \pm 0.2 \mathrm{kHz}$ linewidth (Fig. 2). Initially this analysis addresses the contributions included in Eq. (1), which are represented in Fig. 4. The lifetime of the ${ }^{5} D_{0}$ excited state $(940 \pm 50 \mu$ s) [18] contributes $\Gamma_{\text {pop }}=170 \pm 10 \mathrm{~Hz}$ to the total broadening. The other temperature-independent contribution in Eq. (1) is $\Gamma_{\text {ion-ion, }}$ which manifests as ISD. Although detailed ISD measurements were not performed in this set of experiments, it is possible to place an upper bound on the $\Gamma_{\text {ion-ion }}$ using the results of detailed studies by Thiel et al. [26]. By calculating the excitation density of $\mathrm{Eu}^{3+}, \Gamma_{\text {ion-ion }}$ can be bounded using the ISD coefficient measured for $\mathrm{Eu}^{3+}: \mathrm{Y}_{2} \mathrm{O}_{3}\left(\beta_{\text {ISD }}=1.3 \times 10^{-13} \mathrm{~Hz} /\right.$ (excited ions $\mathrm{cm}^{-3}$ ) [26]. Given the concentration of the sample $(0.5 \%)$, the inhomogeneous linewidth $(19.5 \mathrm{GHz})$ [18], and a maximum excitation bandwidth of $<1 \mathrm{MHz}, \Gamma_{\text {ion-ion }}$ is bounded above by $700 \mathrm{~Hz}$.

The temperature-dependent contributions to the linewidth are $\Gamma_{\mathrm{TLS}}$ and $\Gamma_{\text {phonon. According to the measurements de- }}$ tailed in Sec. III B1, these two broadening mechanisms are characterized by the coefficients $\alpha_{\mathrm{TLS}}=560 \pm 40 \mathrm{~Hz} / \mathrm{K}$ and $\alpha_{\text {phonon }}=0.00088 \pm 0.00005 \mathrm{~Hz} / \mathrm{K}^{7}$. At a temperature of $1.7 \mathrm{~K}$, the temperature at which the magnetic field dependence measurements were conducted, the total broadening contribution is $950 \pm 70 \mathrm{~Hz}$. Therefore, given the current knowledge of the broadening mechanisms in the ceramic sample at $1.7 \mathrm{~K}, 1820 \pm 80 \mathrm{~Hz}$ can confidently be accounted for.

To ascertain whether the mechanism or mechanisms responsible for the remaining $1.4 \mathrm{kHz}$ of broadening were consistent with technical constraints of our apparatus, two-pulse echo measurements were conducted in a $0.02 \% \mathrm{Eu}^{3+}: \mathrm{Y}_{2} \mathrm{SiO}_{5}$ crystal grown for the study in Ref. [58]. In this sample, a linewidth of $740 \pm 10 \mathrm{~Hz}$ was observed at zero magnetic field. In contrast, with an applied field of $400 \mathrm{mT}$, significant spectral diffusion was observed on a time scale of $500 \mu \mathrm{s}$. The broadening with and without an applied field could be increased by approximately $200 \mathrm{~Hz}$ by changing the repetition rate of the combined photon echo and optical repumping sequence from $5 \mathrm{~Hz}$ to $60 \mathrm{~Hz}$.

The results of the system characterization show that the $\Gamma_{h}$ measured in the ceramics are unlikely to be limited by the apparatus. The $60-\mathrm{Hz}$ repetition rate used in the ceramics measurements has been shown to contribute additional broadening of the order of $200 \mathrm{~Hz}$, which we attribute to local heating from the strong optical repumping laser intensity. Although significant, this mechanism is only a fraction of the $1.4 \mathrm{kHz}$ of broadening not yet identified. It is worth noting that because the ceramics are polycrystalline materials, fluctuations in the global environment, for example, magnetic field perturbations due to current noise in the magnet, will cause broadening. The $\mathrm{Eu}^{3+}: \mathrm{Y}_{2} \mathrm{SiO}_{5}$ sample is much less sensitive to such effects because global fluctuations result in a homogeneous phase shift rather than broadening. Global fluctuations are likely to occur on the time scale of $500 \mu \mathrm{s}$, indicated by the spectral diffusion in the $\mathrm{Eu}^{3+}: \mathrm{Y}_{2} \mathrm{SiO}_{5}$ measurements, and hence should not significantly contribute to broadening on the $100-\mu \mathrm{s}$ time scale used for the photon echo measurements of the ceramics. Therefore, the $3.2-\mathrm{kHz}$ broadening observed at $1.7 \mathrm{~K}$ in the post-HIP annealed ceramics is dominated by interactions with TLS, ion-ion interactions in the form of ISD, and a third mechanism intrinsic to the samples. The most likely mechanism is a further magnetic defect, the fluctuations of which are not suppressed at the field magnitudes applied in this work. One possibility is the presence of Kramers rare-earth ions, such as erbium, at a parts per million level, which would account for the extra broadening even at fields of up to $2 \mathrm{~T}$. To probe the material in greater detail to confirm the origin of the remaining broadening, both the temperature and excitation bandwidth should be minimized. This would make the currently unidentified broadening mechanism the dominant perturbation of a total broadening of the order of $1.5 \mathrm{kHz}$. A stable laser possessing a linewidth much less than $1 \mathrm{kHz}$ on a millisecond time scale would provide a large advantage in such a study.

\section{CONCLUSION}

We studied $\mathrm{Eu}^{3+}$-doped $\mathrm{Y}_{2} \mathrm{O}_{3}$ transparent ceramics using temperature- and magnetic-field-dependent two- and threepulse photon echo measurements for the ${ }^{7} F_{0} \leftrightarrow{ }^{5} D_{0}$ transition in order to identify the different dephasing mechanisms that lead to additional broadening of the homogeneous linewidths. In samples that were annealed after the HIP treatment, narrow linewidths of about $3 \mathrm{kHz}$ are demonstrated, which are among the narrowest optical transitions observed in $\mathrm{Eu}^{3+}$-doped materials, including $\mathrm{Eu}^{3+}$-doped $\mathrm{Y}_{2} \mathrm{O}_{3}$ single crystals. We assigned different known broadening contributions and also identified in temperature-dependent measurements a dephasing mechanism that we relate to a magnetic perturbation. An important result is that significant differences in the coherence properties of samples that had undergone different annealing treatment are observed. In this regard, especially the oxygen 
partial pressure seems to play a crucial role in controlling the type and concentration of defects in the anion sublattice. This shows the importance of exactly controlling the synthesis conditions and the understanding of the effects on the final materials, such as the type and concentration of intrinsic defects, but also, for instance, the grain sizes and amount of microstructural strain. Here, the identification of the different broadening contributions will allow an improvement of the the coherence properties in the future by choosing better synthesis conditions. In this way, transparent ceramics could be an attractive alternative to single crystals in quantum memory and spectral filtering applications.

\section{ACKNOWLEDGMENTS}

N.K. would like to thank the Deutsche Forschungsgemeinschaft (DFG) for a postdoctoral research fellowship (Project No. KU 3427/1-1). Financial support by the ANR projects RAMACO (No. 12-BS08-0015-01) and DISCRYS (No. 14CE26-0037-01), Idex ANR-10-IDEX- 0001-02 PSL $\star$, and the Nano'K project RECTUS are gratefully acknowledged. Furthermore, we would like to thank M. O. Ramírez and L. E. Bausá for help with the microscopic measurements, Charles W. Thiel for fruitful discussions, and Jean-François Egrand for constructing a sample holder for the measurements in the high magnetic field.
[1] W. Tittel, M. Afzelius, T. Chanelière, R. L. Cone, S. Kröll, S. A. Moiseev, and M. J. Sellars, Laser Photon. Rev. 4, 244 (2010).

[2] Ph. Goldner, A. Ferrier, and O. Guillot-Noël, in Handbook on the Physics and Chemistry of Rare Earth (North Holland, Amsterdam, 2015), Vol. 46, p. 1.

[3] J.-L. Le Gouët, F. Bretenaker, and I. Lorgeré, Advances in Atomic, Molecular and Optical Physics (Elsevier, New York, 2006), Vol. 54.

[4] M. J. Thorpe, L. Rippe, T. Fortier, M. S. Kirchner, and T. Rosenband, Nat. Photon. 5, 688 (2011).

[5] Y. Li, H. Zhang, C. Kim, K. H. Wagner, P. Hemmer, and L. V. Wang, Appl. Phys. Lett. 93, 011111 (2008).

[6] G. Wolfowicz, H. Maier-Flaig, R. Marino, A. Ferrier, H. Vezin, J. J. L. Morton, and Ph. Goldner, Phys. Rev. Lett. 114, 170503 (2015).

[7] R. M. Macfarlane, J. Lumin. 100, 1 (2002).

[8] T. Böttger, C. W. Thiel, R. L. Cone, and Y. Sun, Phys. Rev. B 79, 115104 (2009).

[9] R. W. Equall, Y. Sun, R. L. Cone, and R. M. Macfarlane, Phys. Rev. Lett. 72, 2179 (1994).

[10] I. Usmani, M. Afzelius, H. de Riedmatten, and N. Gisin, Nat. Commun. 1, 12 (2010).

[11] R. W. Equall, R. L. Cone, and R. M. Macfarlane, Phys. Rev. B 52, 3963 (1995).

[12] M. Macfarlane, Opt. Lett. 18, 1958 (1993).

[13] C. W. Thiel, N. Sinclair, W. Tittel, and R. L. Cone, Phys. Rev. B 90, 214301 (2014).

[14] T. Böttger, C. W. Thiel, Y. Sun, and R. L. Cone, Phys. Rev. B 73, 075101 (2006).

[15] C. W. Thiel, W. R. Babbitt, and R. L. Cone, Phys. Rev. B 85, 174302 (2012).

[16] J. Hegarty, J. Lumin. 36, 273 (1987).

[17] R. M. Macfarlane and R. M. Shelby, J. Lumin. 36, 179 (1987).

[18] N. Kunkel, A. Ferrier, C. W. Thiel, M. O. Ramírez, L. E. Bausá, R. L. Cone, A. Ikesue, and Ph. Goldner, APL Mater. 3, 096103 (2015).

[19] A. Ferrier, C. W. Thiel, B. Tumino, M. O. Ramírez, L. E. Bausá, R. L. Cone, A. Ikesue, and Ph. Goldner, Phys. Rev. B 87, 041102 (2013).

[20] A. Ikesue, T. Kinoshita, K. Kamata, and K. Yoshida, J. Am. Ceram. Soc. 78, 1033 (1995).

[21] V. Lupei, A. Lupei, and A. Ikesue, Appl. Phys. Lett. 86, 111118 (2005).
[22] Y. Sato, J. Saikawa, T. Taira, and A. Ikesue, Opt. Mater. (Amsterdam, Neth.) 29, 1277 (2007).

[23] J. Lu, K. Takaichi, T. Uematsu, A. Shirakawa, M. Musha, K. Ueda, H. Yagi, T. Yanagitani, and A. A. Kaminskii, Appl. Phys. Lett. 81, 4324 (2002).

[24] T. Yanagida, A. Fukabori, Y. Fujimoto, A. Ikesue, K. Kamada, J. Kataoka, Y. Yokota, A. Yoshikawa, and V. Chani, Phys. Status Solidi C 8, 140 (2011).

[25] A. A. Kaminskii, M. S. Akchurin, N. Tanaka, H. J. Eichler, H. Rhee, K. Ueda, T. Takaichi, A. Shirakawa, M. Tokurakawa, J. Dong et al., Phys. Status Solidi A 205, 1666 (2008).

[26] C. W. Thiel, R. M. Macfarlane, Y. Sun, T. Böttger, N. Sinclair, W. Tittel, and R. L. Cone, in Quantum Light-Matter Interaction in Solid State Systems, Barcelona, 2015 (unpublished).

[27] R. M. Macfarlane and R. M. Shelby, Opt. Commun. 39, 169 (1981)

[28] G. P. Flinn, K. W. Jang, J. Ganem, M. L. Jones, R. S. Meltzer, and R. M. Macfarlane, Phys. Rev. B 49, 5821 (1994).

[29] G. P. Flinn, K. W. Jang, J. Ganem, M. L. Jones, R. S. Meltzer, and R. M. Macfarlane, J. Lumin. 58, 374 (1994).

[30] K. S. Hong, R. S. Meltzer, D. K. Williams, and B. M. Tissue, J. Lumin. 76-77, 234 (1998).

[31] A. Perrot, Ph. Goldner, D. Giaume, M. Lovrič, C. Andriamiadamanana, R. R. Gonçalvez, and A. Ferrier, Phys. Rev. Lett. 111, 203601 (2013).

[32] M. Faucher and J. Pannetier, Acta Crystallogr., Sect. B: Struct. Crystallogr. Cryst. Chem. 36, 3209 (1980).

[33] N. Kunkel, J. Bartholomew, L. Binet, A. Ikesue, and Ph. Goldner, J. Phys. Chem. C 120, 13725 (2016).

[34] J. Huang, J. M. Zhang, A. Lezama, and T. W. Mossberg, Phys. Rev. Lett. 63, 78 (1989).

[35] G. K. Liu and R. L. Cone, Phys. Rev. B 41, 6193 (1990).

[36] G. K. Liu, R. L. Cone, M. F. Joubert, B. Jacquier, and J. L. Skinner, J. Lumin. 45, 387 (1990).

[37] C. W. Thiel, R. M. Macfarlane, Y. Sun, T. Böttger, N. Sinclair, W. Tittel, and R. L. Cone, Laser Phys. 24, 106002 (2014).

[38] D. E. McCumber and M. D. Sturge, J. Appl. Phys. 34, 1682 (1963).

[39] W. R. Babbitt, A. Lezama, and T. W. Mossberg, Phys. Rev. B 39, 1987 (1989).

[40] M. M. Broer and B. Golding, J. Lumin. 31-32, 733 (1984).

[41] R. M. Macfarlane, F. Könz, Y. Sun, and R. L. Cone, J. Lumin. 86, 311 (2000). 
[42] K. W. Jang and R. S. Meltzer, Phys. Rev. B 52, 6431 (1995).

[43] B. Herzog and E. L. Hahn, Phys. Rev. 103, 148 (1956).

[44] W. B. Mims, Phys. Rev. 168, 370 (1968).

[45] R. M. Macfarlane and R. M. Shelby, Opt. Commun. 42, 346 (1982).

[46] Y. S. Bai and M. D. Fayer, Phys. Rev. B 39, 11066 (1989).

[47] M. U. Staudt, S. R. Hastings-Simon, M. Afzelius, D. Jaccard, W. Tittel, and N. Gisin, Opt. Commun. 266, 720 (2006).

[48] R. M. Macfarlane, Y. Sun, P. B. Sellin, and R. L. Cone, J. Lumin. 127, 61 (2007).

[49] C. W. Thiel, T. Böttger, and R. L. Cone, J. Lumin. 131, 353 (2011).

[50] J. L. Black and B. I. Halperin, Phys. Rev. B 16, 2879 (1977).

[51] K. A. Littau, M. A. Dugan, S. Chen, and M. D. Fayer, J. Chem. Phys. 96, 3484 (1992).
[52] F. Könz, Y. Sun, C. W. Thiel, R. L. Cone, R. W. Equall, R. L. Hutcheson, and R. M. Macfarlane, Phys. Rev. B 68, 085109 (2003).

[53] K. Chiang, E. A. Whittaker, and S. R. Hartmann, Phys. Rev. B 23, 6142 (1981).

[54] R. M. MacFarlane, R. Wannemacher, D. Boye, Y. P. Wang, and R. S. Meltzer, J. Lumin. 48-49, 313 (1991).

[55] W. Mims, K. Nassau, and J. McGee, Phys. Rev. 123, 2059 (1961).

[56] A. Abragam and B. Bleaney, Electron Paramagnetic Resonance of Transition Ions (Oxford University Press, Oxford, UK, 1970), Chaps. 9, 10.

[57] M. J. Sellars, R. S. Meltzer, P. T. H. Fisk, and N. B. Manson, J. Opt. Soc. Am. B 11, 1468 (1994).

[58] A. Ferrier, B. Tumino, and P. Goldner, J. Lumin. 170, 406 (2016). 